\title{
Triptolide inhibits osteoclast formation, bone resorption, RANKL-mediated NF-қB activation and titanium particle-induced osteolysis in a mouse model
}

\begin{abstract}
Jianbin Huang $^{\# 1}$, Lin Zhou ${ }^{\# 2}$, Huafei Wu ${ }^{\# 2,3}$, Nathan Pavlos ${ }^{3}$, Shek Man Chim ${ }^{2}$, Qian Liu ${ }^{2,4}$, Jinmin Zhao ${ }^{4}$, Wei Xue ${ }^{5}$, Renxiong Tan ${ }^{6}$, Jiming $\mathrm{Ye}^{7}$, Jun $\mathrm{Xu}^{8}$, Estabelle S.M. Ang ${ }^{9}$, Haotian Feng $^{10}$, Jennifer Tickner ${ }^{2}$, Jiake $\mathrm{Xu}^{2^{*}}$, Yue Ding ${ }^{1 *}$
\end{abstract}

${ }^{1}$ Orthopaedic Department, Memorial Hospital of Sun Yat-sen University, Guangzhou, China.

${ }^{2}$ School of Pathology and Laboratory Medicine, The University of Western Australia, Perth, WA 6009, Australia

${ }^{3}$ Centre for Orthopaedic Research, School of Surgery, The University of Western Australia, Perth, WA 6009, Australia

${ }^{4}$ Research Centre for Regenerative Medicine, Department of Orthopaedic Surgery, The First Affiliated Hospital of Guangxi Medical University, Guangxi, China, 530021

${ }^{5}$ Key Laboratory of Biomaterials of Guangdong Higher Education Institutes, Department of Biomedical Engineering, Jinan University, Guangzhou, China, 510632

${ }^{6}$ Institute of Functional Biomolecules, Medical School, Nanjing University, Nanjing China, 210093

${ }^{7}$ Health Innovations Research Institute and School of Health Sciences, RMIT University, Melbourne, VIC 3083, Australia

${ }^{8}$ Research Center for Drug Discovery (RCDD), School of Pharmaceutical Sciences, Sun YatSen University, 132 East Circle at University City, Guangzhou, China, 510006

${ }^{9}$ School of Dentistry, University of Western Australia, Perth, WA 6009, Australia

${ }^{10}$ Program of Nutrition and Bone \& Joint Health, Nestlé R\&D (China) Ltd. Building 5, No. 5

Dijin Road, Haidian District, Beijing, China, 100095

\# equal contributors to this work

* To whom correspondence should be addressed:

Professor Yue Ding, Orthopaedic Department, Memorial Hospital of Sun Yat-sen University, Guangzhou, China. E-mail: dingyue36@126.com; and Professor Jiake Xu, School of Pathology and Laboratory Medicine, The University of Western Australia, Nedlands WA 6009. E-mail jiake.xu@uwa.edu.au.

\section{Running Title: Triptolide inhibits osteolysis}

Keywords: Triptolide, osteoclastogenesis, RANKL, NF-אB, osteolysis, titanium particle 


\begin{abstract}
The RANKL-induced NF- $\mathrm{NB}$ signaling pathway is required for osteoclast formation and function. By screening for compounds that inhibit RANKL-induced NF- $\kappa$ B activation using a luciferase reporter gene assay in RAW264.7 cells, we identified triptolide (PG490), as a candidate compound targeting osteoclast differentiation and osteoclast-mediated osteolysis. Triptolide (PG490) is an active compound of the medicinal herb Tripterygium wilfordii Hook F (TWHF) or Lei Gong Teng with known anti-inflammatory properties. We found that triptolide inhibited osteoclastogenesis and bone resorption, as well as RANKLinduced NF- $\mathrm{KB}$ activities as monitored by luciferase reporter gene assays and the nuclear translocation of p65. In vivo studies showed that triptolide attenuates titanium-induced osteolysis and osteoclast formation in a mouse calvarial model. Considering that drugs which protect against localized bone loss are critically needed for the effective treatment of particle-induced osteolysis, our data suggest that triptolide might have therapeutic potential for the treatment of bone lytic diseases caused by prosthetic wear particles.
\end{abstract}




\section{Introduction}

Osteoclasts are multinucleated, terminally differentiated cells that are responsible for the degradation of mineralized bone matrix (Teitelbaum, 2000). Osteoclasts differentiate from mononuclear cells of the monocyte and macrophage lineage through a process collectively known as osteoclastogenesis (Teitelbaum, 2000). Pivotal to this process is the transcription factor nuclear factor kappa B (NF- $\mathrm{B})$, which plays an important role in both osteoclast differentiation and survival (Boyce et al., 2010,Novack et al., 2003,Ruocco et al., 2005,Xing et al., 2002,Xu et al., 2009). Likewise, the Receptor activator of NF- $\kappa B$ ligand (RANKL) is a key cytokine for osteoclast differentiation, survival and function (Kong et al., 1999, Yasuda et al., 1998). Binding of RANKL to its cognate receptor RANK is essential for osteoclastogenesis, with a disruption in either RANKL or RANK manifesting in severe osteopetrosis due to a lack of osteoclast formation (Brage et al., 2004,Jones et al., 2002,Kong et al., 1999, Yoshitake et al., 2008). Thus, RANKL and its associated signaling cascades serve as potential therapeutic targets for the treatment of osteoclast-related diseases.

Prosthetic wear particles, such as polymethylmethacrylate and titanium particles are involved in the initiation and development of periprosthetic osteolysis that leads to irreversible aseptic loosening of prostheses (Goodman et al., 2006). These particles can stimulate osteoclastmediated bone resorption (Goodman et al., 2006), an action that can promote periprosthetic osteolysis in the bone microenvironment. In addition, both direct and indirect mechanisms of particle-induced osteolysis may involve exacerbated inflammation with the production of pro-inflammatory cytokines, impaired periprosthetic bone formation, and compromised bone regeneration (Wang et al., 2004). To date, effective therapy against particle-induced osteolysis is limited to surgical revision. Identification of drugs that can inhibit particleinduced osteolysis therefore remains a major challenge in the treatment of particle-induced osteolysis.

Triptolide $\left(\mathrm{C}_{20} \mathrm{H}_{24} \mathrm{O}_{6}\right)$ is a highly oxygenated diterpene triepoxide and contains 9,11-epoxide and the 14ß-oriented-hydroxyl system, which are necessary for the anti-leukaemic and immunosuppressive activity (Tao et al., 2001). Triptolide is involved in antitumor activity against prostate cells (Kiviharju et al., 2002), possesses significant therapeutic benefit for immunosuppression (Kusunoki et al., 2004), has anti-inflammatory and anti-leukemia activity (Tao and Lipsky, 2000, Tao et al., 2002), and blocks the induction of NF- $\kappa$ B activity by TNF$\alpha$, through functioning as a NF- $\kappa$ B inhibitor.(Fidler et al., 2003) Previous studies have shown 
that triptolide inhibits NF- $\kappa \mathrm{B}$ transcriptional activation triggered by phorbol 12-myristate 13acetate (PMA), PMA/Iono and TNF- $\alpha$ stimuli via specific binding of the p50-p65 heterodimer to DNA (Qiu et al., 1999). However, no data has been available in regards to whether triptolide exerts a therapeutic effect on particle-induced osteolysis, as well as on RANKL -mediated osteoclastogenesis and NF- $\kappa \mathrm{B}$ activation.

Through the biological screening for compounds that inhibit RANKL-induced NF- $\kappa B$ activation by a luciferase reporter gene assay in RAW264.7 cells, we identified Triptolide (PG490), as a candidate compound targeting osteoclasts and osteoclast-mediated osteolysis. Our results demonstrate that triptolide suppresses osteoclastogenesis, bone resorption and RANKL-mediated NF- $\kappa \mathrm{B}$ activation, and attenuates particle-induced osteolysis in vivo, suggesting that triptolide possesses therapeutic potential for the treatment of osteolysis. 


\section{Materials and Methods}

\subsection{Media and reagents}

RAW264.7 cells were obtained from the American Type Culture Collection (Rockville, MD). $\alpha$-Modification of Eagle's Medium ( $\alpha$-MEM) and fetal bovine serum (FBS) were purchased from TRACE (Sydney, NSW, Australia). L-glutamine, penicillin and streptomycin were purchased from Gibco BRL (Melbourne, Australia). Triptolide was purchased from Axxora Sapphire Bioscience Pty. Ltd. (AU-Redfern, NSW, Australia). Triptolide powder was dissolved in dimethyl sulfoxide (DMSO) and then diluted in phosphate buffered saline (PBS). Natural compounds (with a purity $\geq 98 \%$ ) were obtained from Professor Jun Xu of San Yatsen University, Professor Ren Xiang Tan of Nanjing University, Professor Jiming Ye RMIT University, Melbourne, or Chengdu MUST Bio-technology Co., LTD (China). All compounds were dissolved in dimethysulfulfoxide (DMSO) purchased from BDH laboratory supplies (Poole, Dorset, England). Recombinant GST-RANKL was prepared as previously described (Xu et al., 2000).

\subsection{Drug screening assay and NF-кB activity in RAW264.7 cells}

Drug biological screening assays were conducted to evaluate RANKL-mediated NF- $\kappa \mathrm{B}$ activity using RAW264.7 cells stably transfected with an NF- $\kappa B$-driven luciferase reporter gene construct ( $3 \kappa \mathrm{B}-$ Luc-SV40) (Wang et al., 2003) with natural and synthetic compounds at concentrations of 1 to $10 \mu \mathrm{M}$. To investigate the effect of triptolide on RANKL-induced NF-

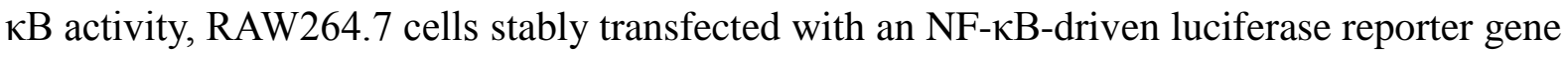

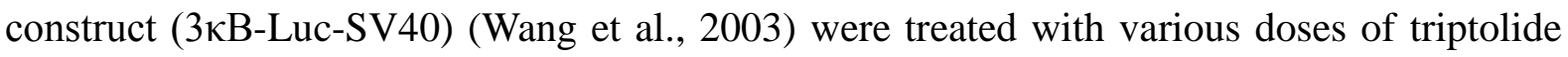
for 1 hour prior to the incubation with 100ng/ml RANKL for 8 hours. Luciferase activities were measured using a POLARstar Optima luminescence detection system (BMG Labtech) (Wang et al., 2003).

\subsection{Osteoclastogenesis assay}

Primary bone marrow macrophages (BMM) isolated from C57BL/6 mice were seeded $\left(1 \times 10^{4}\right.$ cells/well) in a 96-well cell culture plate and incubated overnight with $10 \mathrm{ng} / \mathrm{ml}$ of MCSF before stimulation with RANKL (100 ng/ml) in various concentrations of triptolide. Culture medium was replenished every second day. After 5 days incubation, cells were fixed with $4 \%$ paraformaldehyde and stained for tartrate-resistant acid phosphatase (TRAP) activity. TRAPpositive multinucleated cells with >3 nuclei were scored as osteoclasts (Ang et al., 2009). 


\subsection{Bone resorption pit assay}

To examine the effect of triptolide on osteoclastic-bone resorption, BMM derived osteoclasts cultured from collagen coated plates were cultured in $\alpha$-MEM medium supplemented with $10 \%$ FBS and $100 \mathrm{IU} / \mathrm{ml}$ penicillin, $100 \mu \mathrm{g} / \mathrm{ml}$ streptomycin, $2.5 \mathrm{mg} / \mathrm{ml}$ amphotericin and 50 IU mycostatin at $37^{\circ} \mathrm{C}$ in a water saturated atmosphere with $5 \% \mathrm{CO}_{2}$ and fed by medium replacement every 2-3 days for a total of 5-7 days. Osteoclasts cultured in collagen coated plates were then washed once with PBS and treated for 20 minutes with $250 \mu 1$ of cell dissociation buffer (Sigma) per 25-mm-diameter well. The detached cells were pelleted and resuspended in $150 \mu \mathrm{l}$ of $\alpha$-MEM, aliquoted onto $150 \mu \mathrm{m}$ thick dentine bone slices in wells of a 96 well plate, and incubated for 2 hours to ensure proper attachment of cells onto bone substrate before the addition of Triptolide. After cell attachment, the bone slices were treated either with Triptolide ( $5 \mathrm{nM})$ or left untreated. After 48 hours at $37^{\circ} \mathrm{C}$ incubation, the bone slices were fixed with $4 \%$ paraformaldehyde, osteoclasts were TRAP stained and counted, prior to treatment with $2 \mathrm{M} \mathrm{NaOH}$. Cells were removed by gentle brushing and sonication. The resorption pits were visualized by a Philips XL30 scanning electron microscope (SEM). The percentage of resorbed area on bone surface normalized to osteoclast number was quantified using Scion Image software (Scion Cooperation, National Institute of Health, USA) (Pavlos et al., 2011, Yip et al., 2006).

\section{5 p65 immunohistochemistry}

BMM were seeded $\left(2 \times 10^{4} /\right.$ well in $\left.100 \mu \mathrm{l}\right)$ into 96 -well plates and incubated overnight. Cells were then pre-incubated with triptolide for 1 hour before stimulation with RANKL (100 $\mathrm{ng} / \mathrm{ml}$ ) for up to $30 \mathrm{~min}$. Immunohistochemistry was performed with anti-p65 antibody (Santa Cruz Biotechnology, Inc.) diluted 1:200 in 0.1\% BSA-PBS, and with anti-mouse IgG (Santa Cruz Biotechnology, Inc.) as a negative control as previously described (Ang et al., 2009).

\subsection{Preparation of Titanium Particles}

Commercial pure titanium particles, with a density of $4.507 \mathrm{~g} / \mathrm{cm} 3$, were purchased from Alfa Aesar (Ward Hill, MA, USA). These particles were diluted with pure water and filtered by Millipore filter membranes (Billerica, MA, USA) of a series of sizes (pore diameter: 0.2, 1.2, and $10 \mu \mathrm{m}$ ). Three ranges of particle size, $0.2-1.2 \mu \mathrm{m}$ (TI-0.2), 1.2-10 $\mu \mathrm{m}$ (TI-1.2) and >10 $\mu \mathrm{m}$ (TI-10) were obtained after filtration. All particles were washed with $70 \%$ ethanol for 24 $\mathrm{h}$ at room temperature and then dried in a biological drying oven. The dried particles were 
sterilized with ethylene oxide. According to particle weight and density, the concentration of the particles suspended in PBS was adjusted to $4 \times 10^{8} \mathrm{~mm}^{3} / \mathrm{mL}$. The level of endotoxin in particle solutions was measured using the limulus amebocyte lysate assay (QCl-1000; Bio Whittaker, Walkersville, MD, USA), and the results showed that the endotoxin level was under the detection level of $0.01 \mathrm{EU} / \mathrm{mL}$. An image analysis system (NIKON, Tokyo, Japan) was used to confirm the exact size of the particles, which showed that there were no significant differences in size between different particles (data not shown).

\subsection{Animal Surgery and Experimental Protocol}

Twenty 11-week-old C57BL/6J male mice were purchased from Animal Laboratory of Sun Yat-sen University (Guangzhou, Guangdong, China). All mice were handled in agreement with Chinese and international guidelines for care and use of laboratory animals. Animals were housed in quarantine under local vivarium conditions $\left(24^{\circ} \mathrm{C}, 12 \mathrm{~h} / 12 \mathrm{~h}\right.$ light/dark cycle $)$ for 1 week. The surgical procedures have been described previously.(Wedemeyer et al., 2007) Briefly, mice were operated under general anesthesia via intraperitoneal injection of chloral hydrate. All mice were 12 weeks of age at surgery. A $0.5 \times 0.5 \mathrm{~cm}^{2}$ area of periosteum was exposed by making a $10-\mathrm{mm}$ midline sagittal incision over the calvaria. These mice were divided into 3 groups randomly. Each of five mice received local calvaria injection of $100 \mu \mathrm{l}$ TI-0.2 particles; five mice received $100 \mu 1$ TI-0.2 particles and $50 \mu 1$ triptolide suspension (equivalent to $1 \mathrm{mg} / \mathrm{kg}$ ); five mice sham-operated with $100 \mu \mathrm{l}$ PBS constituted the sham control. The incision was closed using nonabsorbable suture (Yangzhou Guo Tai Co., Ltd, China). The mice were returned to their cages after they were able to right themselves. Water and food were given ad libitum. No mice died during the experiments, and no wound complications were observed. Fourteen days postoperatively, the mice were euthanized, and the calvaria harvested for CT scan analysis and micro-CT imaging.

\subsection{Micro-computed Tomography}

The skulls were analyzed with a high-resolution micro-CT ( $\mu$ CT 80; Scanco Medical AG, Bruettisellen, Switzerland) to perform qualitative analyses of the calvarial bone. Micro-CT analysis focused on the osseous properties in the area of the sagittal suture of the skulls. The radiographic projections $(\mathrm{n}=500)$ were acquired at $55 \mathrm{kV}$ and $145 \mu \mathrm{A}$ with a fixed exposure time of $300 \mathrm{~ms}$ and all projection frames were recorded 6 times and then averaged. 3D images were reconstructed with a voxel average size of $12 \mu \mathrm{m}$, using the manufacturer's reconstruction software (Scanco Medical AG). 


\subsection{Histology}

Paraffin embedded sections $(4 \mu \mathrm{m})$ were taken in the frontal plane centered over the area of particle-induced osteolysis and stained with Goldner dye. The Goldner-stained sections were analyzed by transmission light microscopy (Axiophot, Zeiss, Germany). The existence and dimension of granulomatous foreign body reaction and bone resorption were evaluated using a standard high-quality light microscope. Histomorphometric analysis was made by image analysis software (UTHSCA Image Tool, IT version 3.0; University of Texas, San Antonio, TX) as described previously (Wedemeyer et al., 2007) .

\subsection{Statistical analyses}

Data were presented as mean \pm SEM. Student's t-test was used to determine statistical significance between groups. A p-value of $<0.05$ was considered to be statistically significant. 


\section{Results}

\subsection{Triptolide inhibits osteoclastogenesis in vitro}

Using a high-throughput luciferase reporter gene assay to screen a library of 120 compounds, consisting of natural compounds and their derivatives, for potential inhibitors of RANKLinduced activation of NF- $\kappa \mathrm{B}$, we identified triptolide (Fig. 1), as a candidate compound that potently inhibited RANKL-induced NF- $\kappa B$ activation (Table 1). Given that NF- $\kappa B$ activity is crucial to osteoclast formation and function, we first investigated the cellular and molecular basis of the inhibitory effects of triptolide on osteoclastogenesis in vitro using an established primary bone marrow macrophage (BMM) osteoclastogenic culture system. As shown in Figure 2, mouse BMM cells cultured in M-CSF and RANKL (100 ng/ml) alone formed multinucleated osteoclast-like cells showing typical TRAP positive activity (pink reaction product). On the other hand, BMM cells treated with triptolide demonstrated a dosedependent reduction of RANKL-induced osteoclast formation both in numbers and in size (Fig. 2). In the presence of triptolide, RANKL treatment is still able to induce TRAP expression with smaller size of osteoclast, suggesting that triptolide might affect both the early stage of osteoclast differentiation and the cell fusion at the later stage.

\subsection{Triptolide inhibits osteoclastic bone resorption}

Next, to examine the inhibitory effect of triptolide on osteoclastic bone resorption, a bone resorption pit assay was performed using BMM-derived osteoclasts. As shown in Figure 3A, triptolide $(5 \mathrm{nM})$ suppressed osteoclastic bone resorption activity, as evidenced by the visible reduction in bone resorptive pits by SEM. Quantitative analysis of the total resorption area revealed the area of bone resorption normalized with osteoclast numbers was significantly reduced in osteoclasts treated with triptolide (Fig. 3B) compared with controls treated with vehicle (DMSO) only. These results indicate that triptolide impairs osteoclastic resorptive function in vitro.

\subsection{Triptolide suppresses RANKL-induced activation of NF-қB in osteoclast like cells}

As aforementioned, the NF- $\mathrm{B}$ pathway plays a crucial role in RANKL-induced osteoclastogenesis. Therefore, we next, investigated the effect of triptolide on NF-қB activity using RAW264.7 cells stably transfected with an NF-қB-driven luciferase reporter gene construct ( 3 қВ-Luc-SV40). As shown in Figure 4, RANKL induced a significant increase in NF-қВ activation compared to unstimulated RAW264.7 cells which served as a basal control. Treatment with triptolide showed dose-dependent suppression of RANKL-stimulated NF-қB 
activation in RAW264.7 cells at concentrations of $0.62 \mathrm{nM}, 1.25 \mathrm{nM}$ and $2.5 \mathrm{nM}(\mathrm{p}<0.001)$.

\subsection{Triptolide suppresses RANKL-induced p65 nuclear translocation}

To further explore the effect of triptolide on NF- $\kappa \mathrm{B}$ activity, we evaluated the effect of triptolide on RANKL-mediated p65 nuclear translocation in BMMs. Figure 5 shows that treatment of BMMs with triptolide decreases the RANKL-induced cytosol-nuclear translocation of $\mathrm{p} 65$, as compared to vehicle control cells which possessed a higher percentage of cells displaying clear nuclear translation of p65. Anti-mouse IgG, which served as a negative control, showed negligible staining (Fig. 5).

\subsection{Triptolide treatment reduced titanium particle-induced osteolysis of calvarial bone.}

Next, we examined the effect of triptolide on titanium particle-induced osteolysis in vivo using our previously published procedures (Wedemeyer et al., 2007). During the treatment, we did not observe weight loss and abnormal behavior of triptolide treated mice. Micro-CT analysis showed that administration of titanium particles to the surface of mouse calvarial bone elicits a localized inflammatory and osteolytic response which significantly widens the cranial suture width. By comparison, Sham-operated mice show no obvious signs of osteolytic activity. Importantly, treatment with triptolide significantly decreased the cranial suture width induced by titanium particles (Fig. 6).

To enable more detailed examination of the effects of triptolide on sagittal suture resorption area and osteoclast formation in vivo, we performed histological assessment of the calvaria. As shown in Figure 6C, titanium particles caused a significant increase in sagittal suture resorption area, whereas co-injection with triptolide significantly reduced titanium particle-induced sagittal suture resorption area. Taken together, these findings indicate that triptolide attenuates osteoclast-mediated osteolysis. 


\section{Discussion}

In the present study, we addressed the therapeutic potential of triptolide as a candidate inhibitor of osteoclastogenesis, bone resorption, RANKL-induced NF-қB signaling pathways and particle-induced osteolysis. Previous studies have shown that triptolide specifically targets NF-қB p65 to block NF-қB signaling via inhibiting NF-қB transcriptional activation through the C-terminal portion of NF-қB p65 in T-cells (Qiu et al., 1999). Dai et al also revealed that triptolide inhibited the binding of $\mathrm{NF}-\mathrm{kB}$ p65/p50 in COX-2 promoters in lipopolysaccharide (LPS)-stimulated human A172 cells (Dai et al., 2006). Similarly, Zhu et al reported that triptolide inhibits NF-қB transcriptional activity via blocking the association of p65 with CREB-binding protein (CBP)/p300 and via decreasing the p65 protein expression (Zhu et al., 2009). Consistent with these observations, our studies provide further evidence that triptolide efficiently impairs $\mathrm{NF}-\mathrm{kB}$ activity and p65 nuclear translocation during RANKL-induced osteoclastogenesis.

Prosthetic wear particles play a pathological role in the initiation and development of periprosthetic osteolysis, leading to irreversible aseptic loosening of prostheses (Goodman et al., 2006). Polymethylmethacrylate and titanium particles have been commonly associated with clinical aseptic joint loosening and over-activation of osteoclasts (Goodman et al., 2006). Many orthopaedic implants fail due to particle-stimulated aseptic loosening. To date, therapy against particle-induced osteolysis is limited to surgical revision. Thus, identification of drugs that can suppress particle-induced osteolysis will improve treatment options for particlestimulated aseptic loosening. In this study, we demonstrate that triptolide effectively suppressed titanium particle-induced osteolysis. Osteolysis induced by titanium particles is accompanied by increased numbers of osteoclasts locally in mice. Interestingly, we found that triptolide treatment reduced osteoclast formation in titanium particle-treated animals in vivo, and RANKL-mediated osteoclastogenesis in vitro, an action that has not been previously assigned to this compound. Based on our in vitro observations, it is likely that the direct inhibition of RANKL-induced osteoclastogenesis by triptolide largely accounts for the suppression of titanium particle-induced osteolysis observed in vivo.

Triptolide has also been shown to display a therapeutic effect on solid tumor, leukemia, and a variety of inflammatory disorders, which is often accompanied by increased osteoclast formation (Chen, 2001, Wang et al., 2008, Yang et al., 2003, Yao et al., 2008). Some preclinical in vivo and in vitro studies report that triptolide delays onset of arthritis, lowers the 
arthritis scores and reduces the expression of osteoclast-activating factors ( $\mathrm{Hu}$ et al., 2006,Matta et al., 2009,Xiao et al., 2009). Our results suggest that triptolide at a dose of 1 $\mathrm{mg} / \mathrm{kg}$ is effective to reduce titanium particles-induced osteolysis locally. The therapeutic effects of triptolide on osteolysis observed in this study are in good accord with previous studies employing selective modulators of NF-қB for anti-osteoclast activity through the interruption of RANKL-mediated NF-қB signaling pathways (Boyce et al., 2010,Novack et al., 2003,Ruocco et al., 2005,Xing et al., 2002,Xu et al., 2009).

It is worth mentioning that, in addition to its growing list of therapeutic benefits, triptolide also exhibits adverse side effects with occasional severe toxicity. Many in vitro studies show the anti-inflammatory effects of triptolide at low doses, for example, in multiple myeloma cells at concentrations of 28-224 nM (Lou et al., 2005), in bronchial epithelial cells (Zhao et al., 2000) and in T-cells (Qiu et al., 1999) at concentrations of $\geq 56 \mathrm{nM}$, and in lipopolysaccharide-stimulated macrophages at doses of 10-50 nM (Matta et al., 2009). With respect to these reports, our results demonstrate that triptolide significantly inhibited RANKL-induced osteoclastogenesis at comparatively lower doses which may circumvent toxicity problems when administered in vivo. In this study, it was noted that different concentrations of triptolide were observed on the inhibitory effects of NF- $\kappa \mathrm{B}$ luciferase activity, osteoclast formation, and bone resorption, which was most likely due to the exposure of different time courses and sensitivity of various assay systems available. For instance, lower concentration of triptolide was required to inhibit osteoclast formation probably owing to its longer incubation time than that in the NF- $\kappa \mathrm{B}$ reporter gene assay and in the p65 translocation experiments.

In summary, through biological screening of a compound library, we identified triptolide as a potent suppressor of osteoclast formation, bone resorption and RANKL-induced NF-қB activation in vitro, and titanium particle-induced osteolysis in vivo. Together, these results highlight the therapeutic potential of triptolide for treatment of bone lytic diseases. 


\section{Disclosure}

All authors state that they have no conflicts of interest.

\section{Acknowledgements}

This study was supported in part by the National Health and Medical Research Council of Australia, Arthritis Foundation of Western Australia, and University of Western Australia Research Collaboration Awards. This work was also supported by funding from the National Natural Science Foundation of China (No. 81272020 and No. 81228013). Dr. Qian Liu has been a Visiting Scholar to the University of Western Australia. Prof. Jiake Xu was a visiting Professor to the Research Centre for Regenerative Medicine, Guangxi Medical University in 2013. The authors also acknowledge technical suggestions from Dr. An Qin, Jay Steer, Jacob Kenny, Dian Teguh and Prof. Ming Zheng, and equipment operation assistance from the Centre for Microscopy, Characterisation and Analysis (CMCA) of The University of Western Australia. 


\section{Reference}

[1] Teitelbaum, S.L., 2000. Bone resorption by osteoclasts, Science. 289, 1504-8.

[2] Boyce, B.F., Yao, Z. and Xing, L., 2010. Functions of nuclear factor kappaB in bone, Ann N Y Acad Sci. 1192, 367-75.

[3] Novack, D., Yin, L., Hagen-Stapleton, A., Schreiber, R., Goeddel, D., Ross, F. and Teitelbaum, S., 2003. The IkappaB function of NF-kappaB2 p100 controls stimulated osteoclastogenesis, J Exp Med. 198, 771-81.

[4] Ruocco, M., Maeda, S., Park, J., Lawrence, T., Hsu, L., Cao, Y., Schett, G., Wagner, E. and Karin, M., 2005. I $\{$ kappa $\}$ B kinase (IKK) \{beta $\}$, but not IKK $\{$ alpha $\}$, is a critical mediator of osteoclast survival and is required for inflammation-induced bone loss, J Exp Med. . 201, 1677-87.

[5] Xing, L., Bushnell, T., Carlson, L., Tai, Z., Tondravi, M., Siebenlist, U., Young, F. and Boyce, B., 2002. NF-kappaB p50 and p52 expression is not required for RANK-expressing osteoclast progenitor formation but is essential for RANK- and cytokine-mediated osteoclastogenesis, J Bone Miner Res. 17, 1200-10.

[6] Xu, J., Wu, H., Ang, E., Yip, K., Woloszyn, M., Zheng, M. and Tan, R., 2009. NF-kappaB modulators in osteolytic bone diseases, Cytokine Growth Factor Rev. 20, 7-17.

[7] Kong, Y., Yoshida, H., Sarosi, I., Tan, H., Timms, E., Capparelli, C., Morony, S., Oliveira-dos-Santos, A., Van, G., Itie, A., Khoo, W., Wakeham, A., Dunstan, C., Lacey, D., Mak, T., Boyle, W. and Penninger, J., 1999. OPGL is a key regulator of osteoclastogenesis, lymphocyte development and lymph-node organogenesis, Nature 397, 315-23.

[8] Yasuda, H., Shima, N., Nakagawa, N., Yamaguchi, K., Kinosaki, M., S., M., Tomoyasu, A., Yano, K., Goto, M., Murakami, A., Tsuda, E., Morinaga, T., Higashio, K., Udagawa, N., Takahashi, N. and Suda, T., 1998. Osteoclast differentiation factor is a ligand for osteoprotegerin/osteoclastogenesis-inhibitory factor and is identical to TRANCE/RANKL, Proc Natl Acad Sci U S A. 95.

[9] Brage, M., Lie, A., Ransjö, M., Kasprzykowski, F., Kasprzykowska, R., Abrahamson, M., Grubb, A. and Lerner, U., 2004. Osteoclastogenesis is decreased by cysteine proteinase inhibitors, Bone. 34, 41224.

[10] Jones, D., Kong, Y. and Penninger, J., 2002. Role of RANKL and RANK in bone loss and arthritis, Ann Rheum Dis. 61 Suppl 2, ii32-9.

[11] Yoshitake, F., Itoh, S., Narita, H., Ishihara, K. and Ebisu, S., 2008. J Biol Chem. . 283, 11535-40.

[12] Goodman, S.B., Ma, T., Chiu, R., Ramachandran, R. and Smith, R.L., 2006. Effects of orthopaedic wear particles on osteoprogenitor cells, Biomaterials. 27, 6096-101.

[13] Wang, M.L., Sharkey, P.F. and Tuan, R.S., 2004. Particle bioreactivity and wear-mediated osteolysis, J Arthroplasty. 19, 1028-38.

[14] Tao, X., Cush, J., Garret, M. and Lipsky, P., 2001. A phase I study of ethyl acetate extract of the chinese antirheumatic herb Tripterygium wilfordii hook F in rheumatoid arthritis, J Rheumatol. 28, 2160-7.

[15] Kiviharju, T., Lecane, P., Sellers, R. and Peehl, D., 2002. Antiproliferative and proapoptotic activities of triptolide (PG490), a natural product entering clinical trials, on primary cultures of human prostatic epithelial cells, Clin Cancer Res. 8, 2666-74.

[16] Kusunoki, N., Yamazaki, R., Kitasato, H., Beppu, M., Aoki, H. and Kawai, S., 2004. Triptolide, an active compound identified in a traditional Chinese herb, induces apoptosis of rheumatoid synovial fibroblasts, BMC Pharmacol. 4, 2.

[17] Tao, X. and Lipsky, P., 2000. The Chinese anti-inflammatory and immunosuppressive herbal remedy Tripterygium wilfordii Hook F, Rheum Dis Clin North Am. 26, 29-50, viii.

[18] Tao, X., Younger, J., Fan, F., Wang, B. and Lipsky, P., 2002. Benefit of an extract of Tripterygium Wilfordii Hook F in patients with rheumatoid arthritis: a double-blind, placebo-controlled study, Arthritis Rheum. 46, 1735-43.

[19] Fidler, J., Li, K., Chung, C., Wei, K., Ross, J., Gao, M. and Rosen, G., 2003. PG490-88, a derivative of triptolide, causes tumor regression and sensitizes tumors to chemotherapy, Mol Cancer Ther. 2, 855-62.

[20] Qiu, D., Zhao, G., Aoki, Y., Shi, L., Uyei, A., Nazarian, S., Ng, J. and Kao, P., 1999. Immunosuppressant PG490 (triptolide) inhibits T-cell interleukin-2 expression at the level of purinebox/nuclear factor of activated T-cells and NF-kappaB transcriptional activation, J Biol Chem. 274, 13443-50.

[21] Xu, J., Tan, J., Huang, L., Gao, X., Laird, R., Liu, D., Wysocki, S. and Zheng, M., 2000. Cloning, sequencing, and functional characterization of the rat homologue of receptor activator of NF-kappaB ligand, J Bone Miner Res. . 15, 2178-86.

[22] Wang, C., Steer, J.H., Joyce, D.A., Yip, K.H., Zheng, M.H. and Xu, J., 2003. 12-Otetradecanoylphorbol-13-acetate (TPA) inhibits osteoclastogenesis by suppressing RANKL-induced NF-kappaB activation, J Bone Miner Res. 18, 2159-68. 
[23] Ang, E., Pavlos, N.J., Rea, S.L., Qi, M., Chai, T., Walsh, J.P., Ratajczak, T., Zheng, M.H. and Xu, J., 2009. Proteasome inhibitors impair RANKL-induced NF-kappaB activity in osteoclast-like cells via disruption of p62, TRAF6, CYLD, and IkappaBalpha signaling cascades, J Cell Physiol. 220, 450-9.

[24] Pavlos, N.J., Cheng, T.S., Qin, A., Ng, P.Y., Feng, H.T., Ang, E.S., Carrello, A., Sung, C.H., Jahn, R., Zheng, M.H. and Xu, J., 2011. Tctex-1, a novel interaction partner of Rab3D, is required for osteoclastic bone resorption, Mol Cell Biol. 31, 1551-64.

[25] Yip, K., Feng, H., Pavlos, N., Zheng, M. and Xu, J., 2006. p62 ubiquitin binding-associated domain mediated the receptor activator of nuclear factor-kappaB ligand-induced osteoclast formation: a new insight into the pathogenesis of Paget's disease of bone, Am J Pathol 169, 503-14.

[26] Wedemeyer, C., Xu, J., Neuerburg, C., Landgraeber, S., Malyar, N.M., von Knoch, F., Gosheger, G., von Knoch, M., Loer, F. and Saxler, G., 2007. Particle-induced osteolysis in three-dimensional microcomputed tomography, Calcif Tissue Int. 81, 394-402.

[27] Dai, Y., Jin, D., Zhu, X. and Lei, D., 2006. Triptolide inhibits COX-2 expression via NF-kappa B pathway in astrocytes, Neurosci Res. 55, 154-60.

[28] Zhu, W., Ou, Y., Li, Y., Xiao, R., Shu, M., Zhou, Y., Xie, J., He, S., Qiu, P. and Yan, G., 2009. A smallmolecule triptolide suppresses angiogenesis and invasion of human anaplastic thyroid carcinoma cells via down-regulation of the nuclear factor-kappa B pathway, Mol Pharmacol. . 75, 812-9.

[29] Chen, B., 2001. Triptolide, a novel immunosuppressive and anti-inflammatory agent purified from a Chinese herb Tripterygium wilfordii Hook F, Leuk Lymphoma. 42, 253-65.

[30] Wang, X., Liang, X., Li, F., Zhou, H., Liu, X., Wang, J. and Wang, X., 2008. Therapeutic strategies for Parkinson's disease: the ancient meets the future--traditional Chinese herbal medicine, electroacupuncture, gene therapy and stem cells, Neurochem Res. 33, 1956-63.

[31] Yang, S., Chen, J., Guo, Z., Xu, X., Wang, L., Pei, X., Yang, J., Underhill, C. and Zhang, L., 2003. Triptolide inhibits the growth and metastasis of solid tumors, Mol Cancer Ther. 2, 65-72.

[32] Yao, G., Luan, J., Ye, D., Yan, J., Lei, Q., Zhu, P. and Jin, J., 2008. Effects of triptolide on proliferation and apoptosis of jurkat cell line in acute T lymphocytic leukemia, Zhongguo Shi Yan Xue Ye Xue Za Zhi. 16, 506-9.

[33] Hu, Y., Luo, B., Zhang, M., Tu, S. and Zeng, K., 2006. Effect of triptolide on expression of receptor activator of nuclear factor-kappaB ligand in rat adjuvant induced arthritis, J Huazhong Univ Sci Technolog Med Sci. 26, 344-6.

[34] Matta, R., Wang, X., Ge, H., Ray, W., Nelin, L. and Liu, Y., 2009. Triptolide induces anti-inflammatory cellular responses, Am J Transl Res. . 1, 267-82.

[35] Xiao, C., Zhou, J., He, Y., Jia, H., Zhao, L., Zhao, N. and Lu, A., 2009. Effects of triptolide from Radix Tripterygium wilfordii (Leigongteng) on cartilage cytokines and transcription factor NF-kappaB: a study on induced arthritis in rats, Chin Med. 4:13.

[36] Lou, Y., Jin, J. and Wang, Y., 2005. Triptolide inhibits transcription factor NF-kappaB and induces apoptosis of multiple myeloma cells, Leuk Res. . 29, 99-105.

[37] Zhao, G., Vaszar, L., Qiu, D., Shi, L. and Kao, P., 2000. Anti-inflammatory effects of triptolide in human bronchial epithelial cells, Am J Physiol Lung Cell Mol Physiol. . 279, L958-66. 


\section{Figure Captions}

Fig. 1. Overview of the chemical structure of natural compounds and their derivatives used for initial screening and identification of potential NF- $\kappa \mathrm{B}$ modulators. YC4-2 is a tetracyclic terpenoid derivative of oleanic acid. LH8, 13, 16, 22, 32 and 45 are derivatives of Berberine. HuM8 and 13 are derivatives of matrine.

Fig. 2. Triptolide inhibits RANKL-induced osteoclastogenesis. (A) Chemical structure of triptolide. (B) BMMs cultured in a 96-well plate in the presence of RANKL (100ng/ml) and M-CSF (10 ng/ml) with or without triptolide for 5 days were fixed with $4 \%$ paraformaldehyde and stained for TRAP activity. (C) Representative light microscope images showing the effect of triptolide on RANKL-induced osteoclast formation with morphological changes in comparison with BMM cells cultured in the absence of RANKL. (D) Quantitative analysis shows the mean number of TRAP-positive multinucleated cells (MNC). (** $\mathrm{p}<0.01$, *** $\mathrm{p}<0.001$ compared to RANKL treated control, $\mathrm{n}=3$ ).

Fig. 3. Triptolide suppresses osteoclastic bone resorption. BMM derived osteoclasts were seeded on bone slices in the presence and absence of Triptolide ( 0 and $5 \mathrm{nM})$ cultured for 48 hours at $37^{\circ} \mathrm{C}$. (A) Representative SEM images of bone resorption. (B) Resorption area expressed as a percentage of total bone area normalized with osteoclast numbers $(* * *$ $\mathrm{p}<0.001$ compared to control, $\mathrm{n}=3$ ).

Fig. 4. Triptolide suppresses RANKL-induced NF-қВ-dependent transcription in RAW264.7 cells which were stably transfected with the $3 \kappa \mathrm{B}-\mathrm{luc}-\mathrm{SV} 40$ reporter gene, and treated with various doses of triptolide in the absence or presence of $100 \mathrm{ng} / \mathrm{ml}$ RANKL. $(* * * \mathrm{p}<0.001$ compared to RANKL-stimulated control, $n=3$ ).

Fig. 5. Triptolide inhibits RANKL-induced p65 nuclear translocation. (A,B) Treatment with RANKL induces p65 translocation from the cytoplasm to the nucleus in BMM cells. RANKL-induced nuclear translocation was inhibited by pre-treatment with triptolide. (D) Negative control cells treated as in (B), and stained with anti-mouse IgG antibody. Black arrow indicates the localization of p65 in the cytoplasm, and white arrow specifies the presence of p65 in the nucleus. (E) The percentage of cells showing nuclear translocation of p65 was reduced in cells treated with RANKL + triptolide $(5 \mathrm{~nm}$ ) compared to RANKL only 
controls. $(* * * \mathrm{p}<0.001, \mathrm{n}=3)$.

Fig. 6. Effects of triptolide on osteolysis in the cranial suture width induced by titanium particles. (A) Representative images from a high-resolution micro-CT of radiographic projections of the osseous properties in the sagittal suture of the skulls. (TI represents titanium particles; TI + Trip represents titanium particles with triptolide). (B) Treatment with triptolide reduced titanium particle- induced suture widening. (C) Treatment with triptolide reduced titanium particle-induced sagittal suture resorption area. $(* p<0.05, n=5)$. 


\section{Highlights}

Triptolide inhibited osteoclastogenesis and bone resorption, and RANKL-induced NF-kB activities.

Triptolide attenuates titanium-induced osteolysis and osteoclast formation in a mouse calvarial model.

Triptolide might have therapeutic potential for the treatment of bone lytic diseases caused by prosthetic wear particles. 
Table 1: Compound screening of NF-қB inhibitory effect

\begin{tabular}{|c|c|c|}
\hline Compounds name & Origin & $\begin{array}{l}\text { Inhibitory effect on RANKL-induced } \\
\text { NF-KB using luciferase assay (IC50) }\end{array}$ \\
\hline Triptolide & Natural & $2.5 \mathrm{nM}$ \\
\hline Arbiflorin & Natural & No effect or $>10 \mu \mathrm{M}$ \\
\hline Berberine & Natural & $2 \mu \mathrm{M}$ \\
\hline LH8 (Berberin derivatives) & Natural derivatives & No effect or $>10 \mu \mathrm{M}$ \\
\hline LH13 (Berberin derivatives) & Natural derivatives & No effect or $>10 \mu \mathrm{M}$ \\
\hline LH16 (Berberin derivatives) & Natural derivatives & No effect or $>10 \mu \mathrm{M}$ \\
\hline LH22 (Berberin derivatives) & Natural derivatives & No effect or $>10 \mu \mathrm{M}$ \\
\hline LH32 (Berberin derivatives) & Natural derivatives & No effect or $>10 \mu \mathrm{M}$ \\
\hline LH45 (Berberin derivatives) & Natural derivatives & No effect or $>10 \mu \mathrm{M}$ \\
\hline HuM8 (matrine derivatives) & Natural derivatives & No effect or $>10 \mu \mathrm{M}$ \\
\hline HuM13 (matrine derivatives) & Natural derivatives & No effect or $>10 \mu \mathrm{M}$ \\
\hline Paeoniflorin & Natural & No effect or $>10 \mu \mathrm{M}$ \\
\hline Oleanic acid & Natural & No effect or $>10 \mu \mathrm{M}$ \\
\hline YC4-2 & Natural derivatives & No effect or $>10 \mu \mathrm{M}$ \\
\hline Polydatin & Natural & No effect or $>10 \mu \mathrm{M}$ \\
\hline Matrine & Natural & No effect or $>10 \mu \mathrm{M}$ \\
\hline Rotundine & Natural & No effect or $>10 \mu \mathrm{M}$ \\
\hline Arbutin & Natural & No effect or $>10 \mu \mathrm{M}$ \\
\hline Piperine & Natural & No effect or $>10 \mu \mathrm{M}$ \\
\hline
\end{tabular}


Click here to download high resolution image

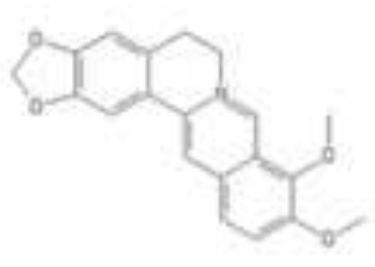

Berberine

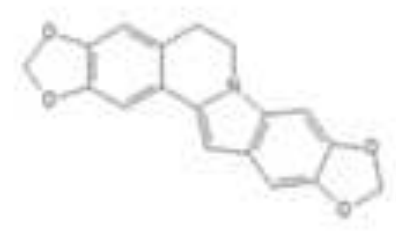

LH45

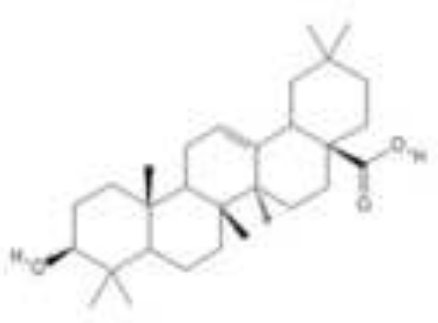

Oleanic acid

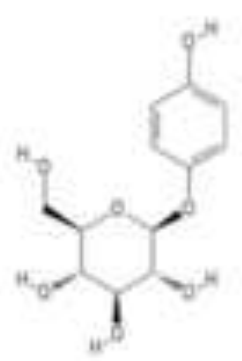

Arbutin

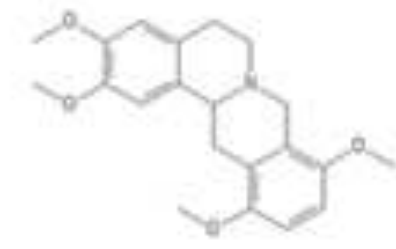

LH8

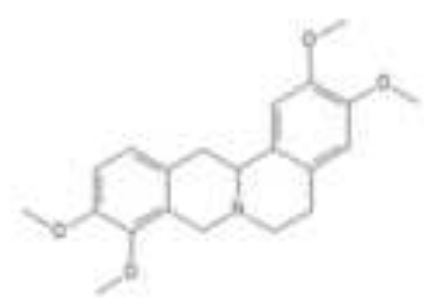

Rotundine
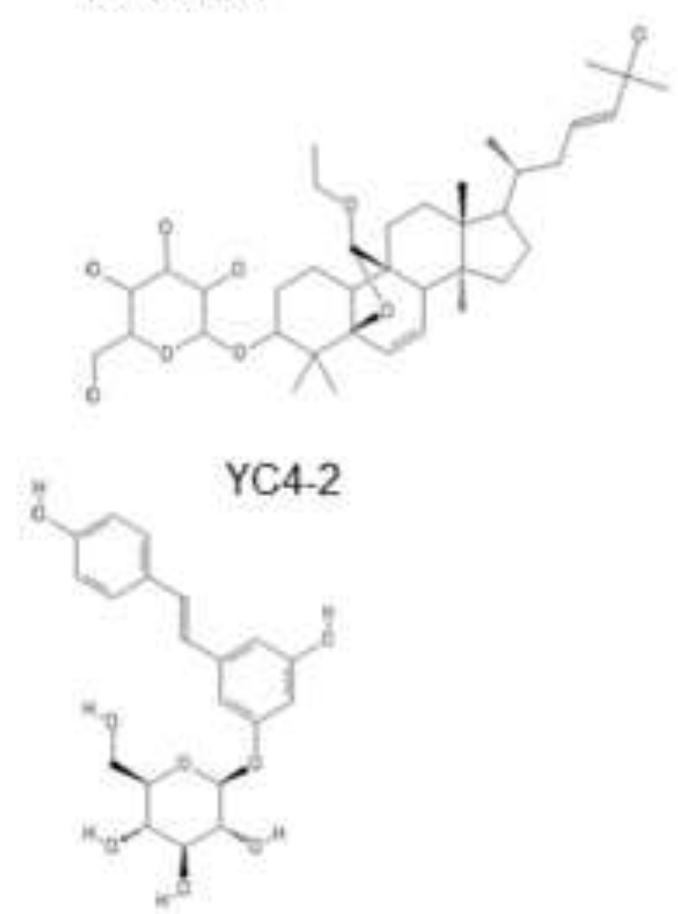

Polydatin

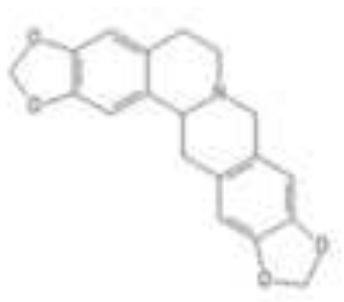

LH16

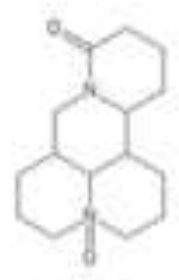

HuM8

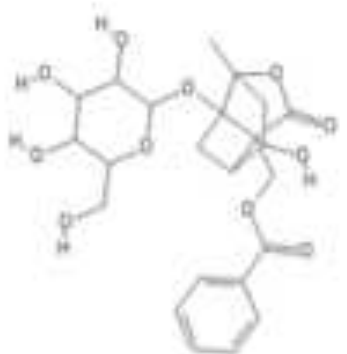

Arbiflorin

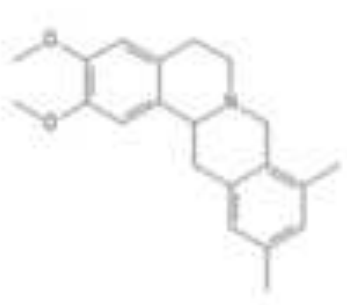

LH22

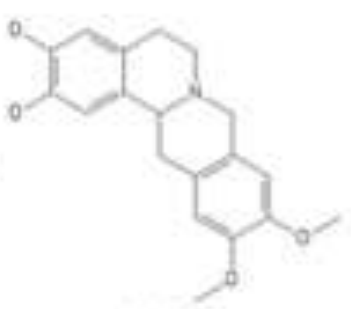

LH32

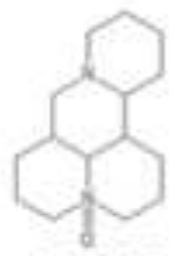

HuM13

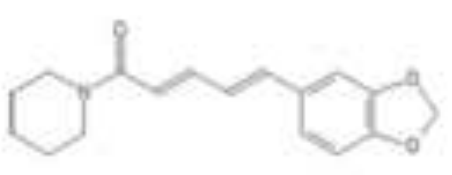

Piperine

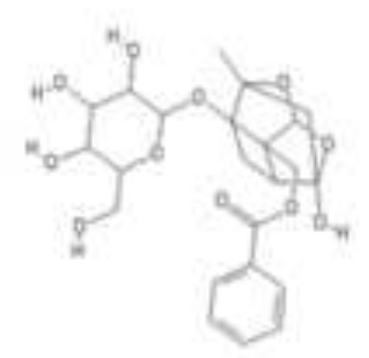

Paeoniflorin

Fig. 1 
A

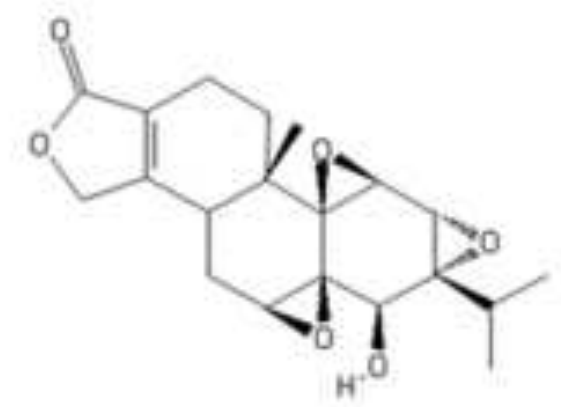

C

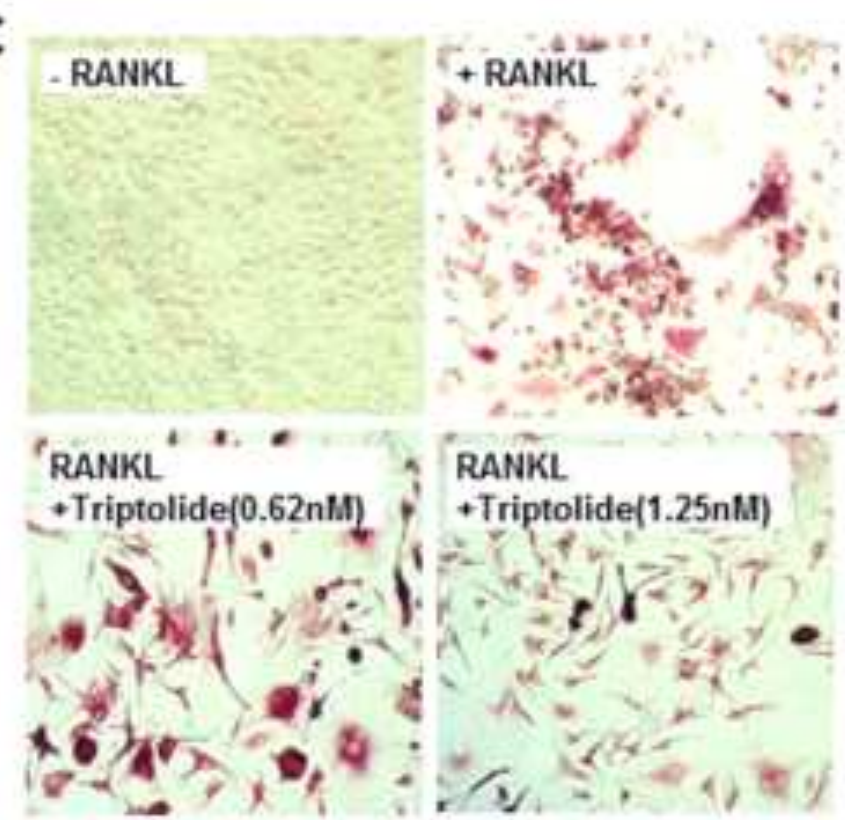

B

RANKL - + + + + + +

Triptolide(nM) $0 \quad 0^{0^{3}} \quad 0^{0^{4}} \quad 0^{5} \quad 0^{3} \quad 0^{6^{2}} \quad 2^{5}$

D

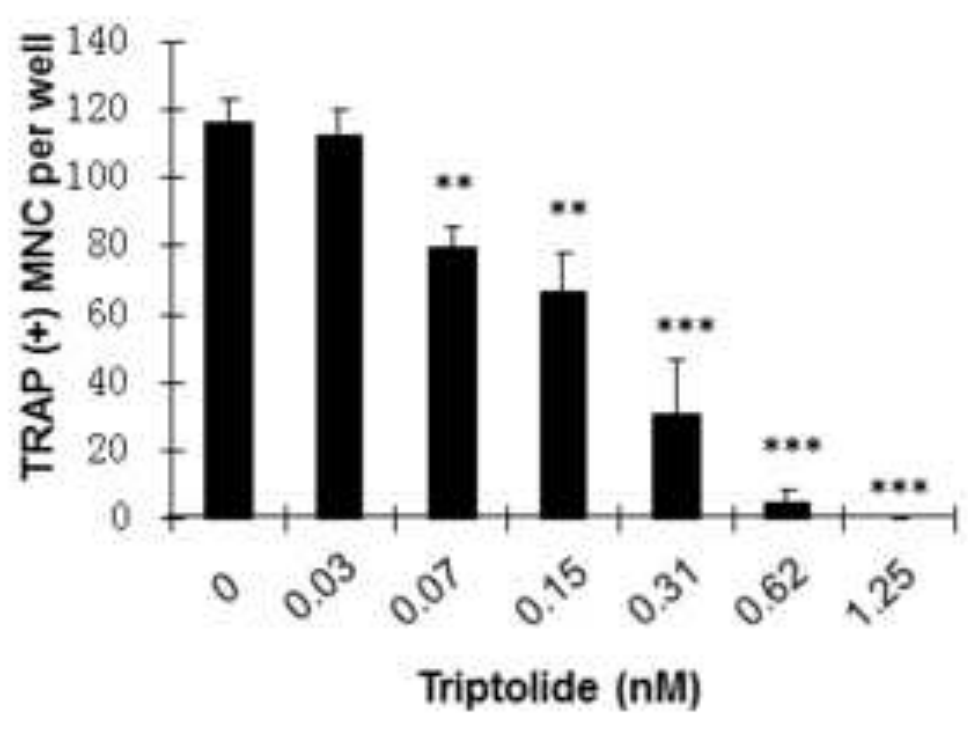

Fig. 2 
Click here to download high resolution image
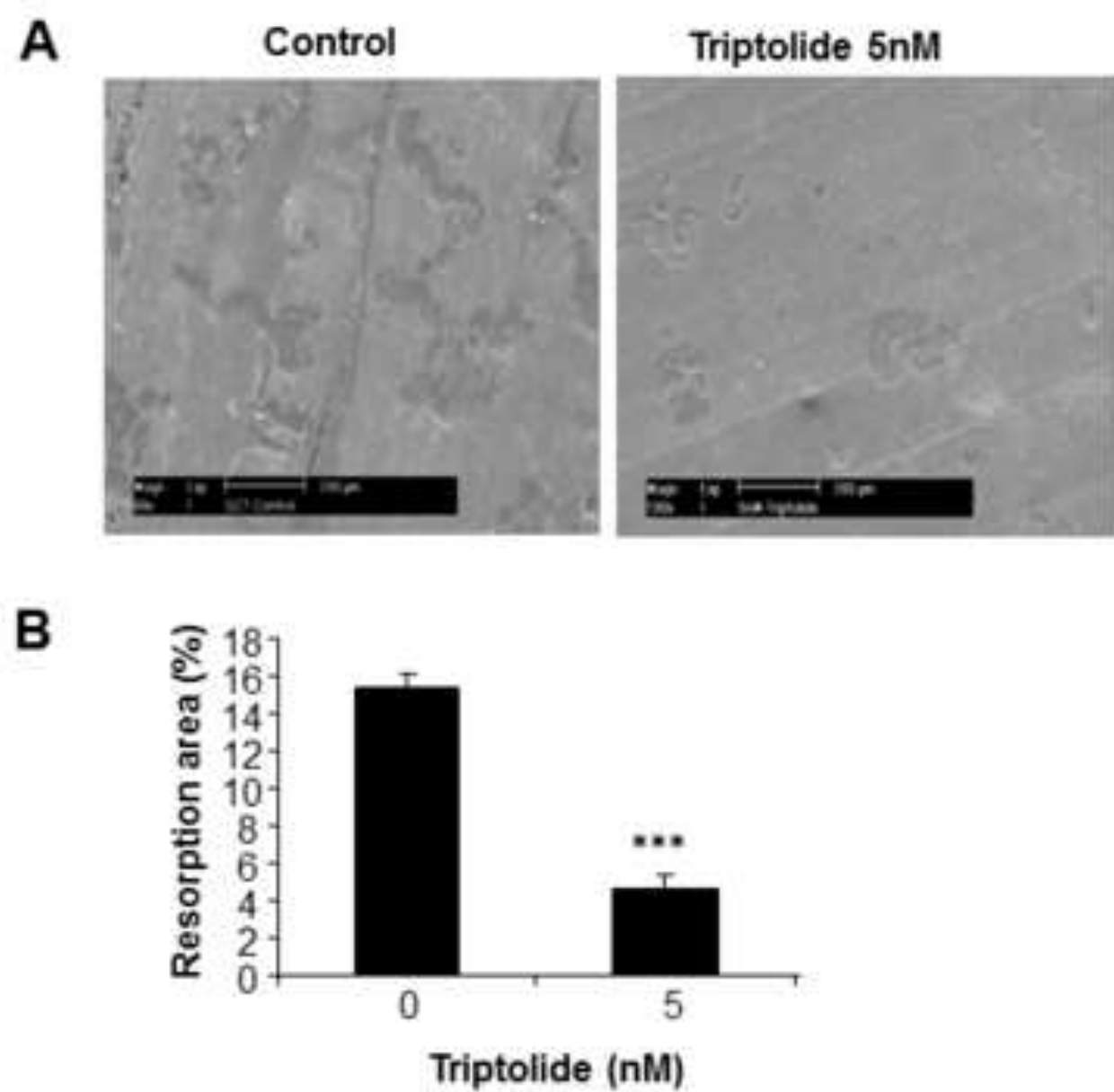

Fig. 3 


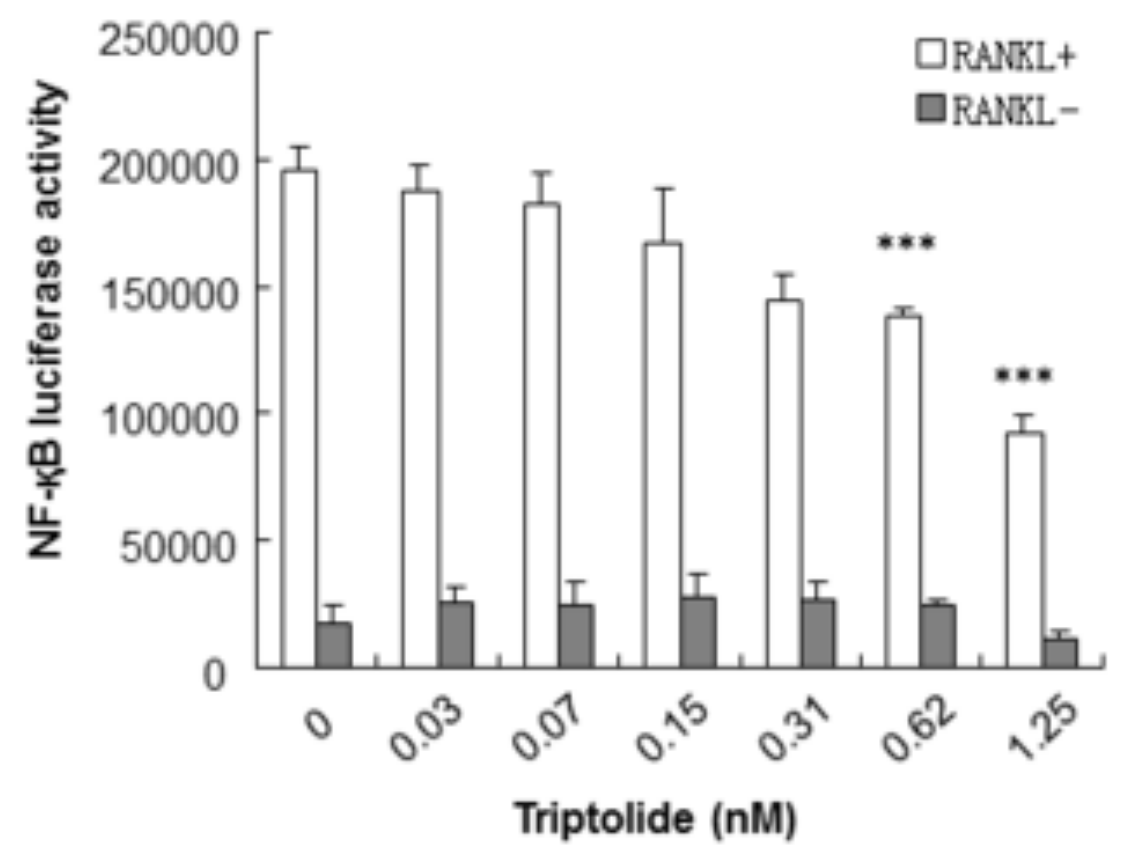

Fig. 4 
A

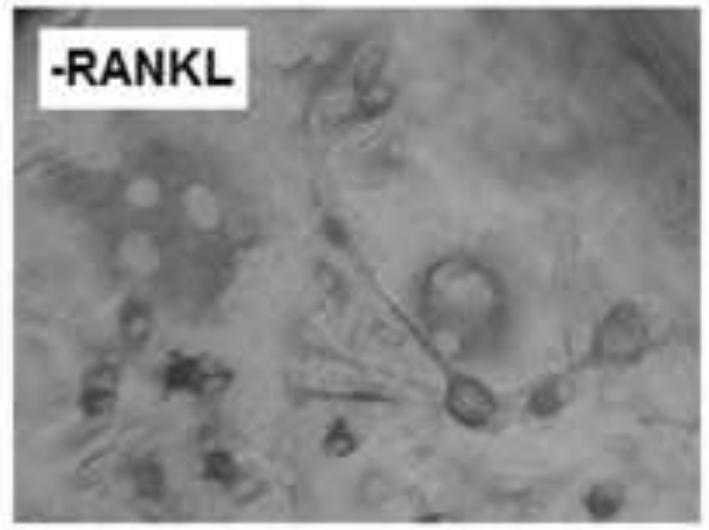

C

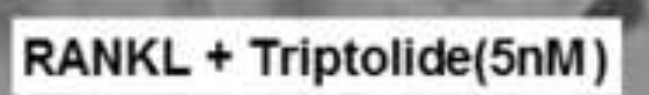

B

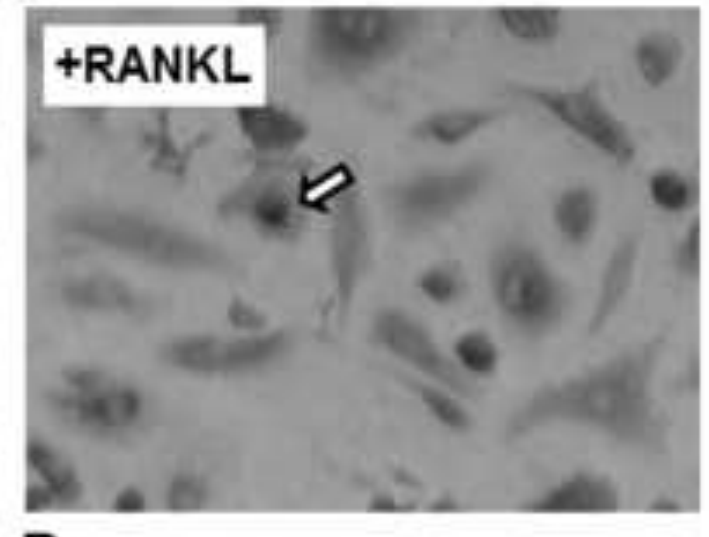

D

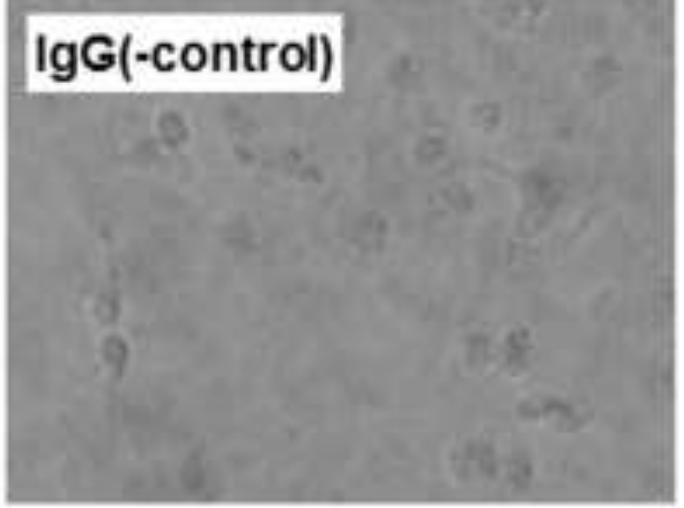

E

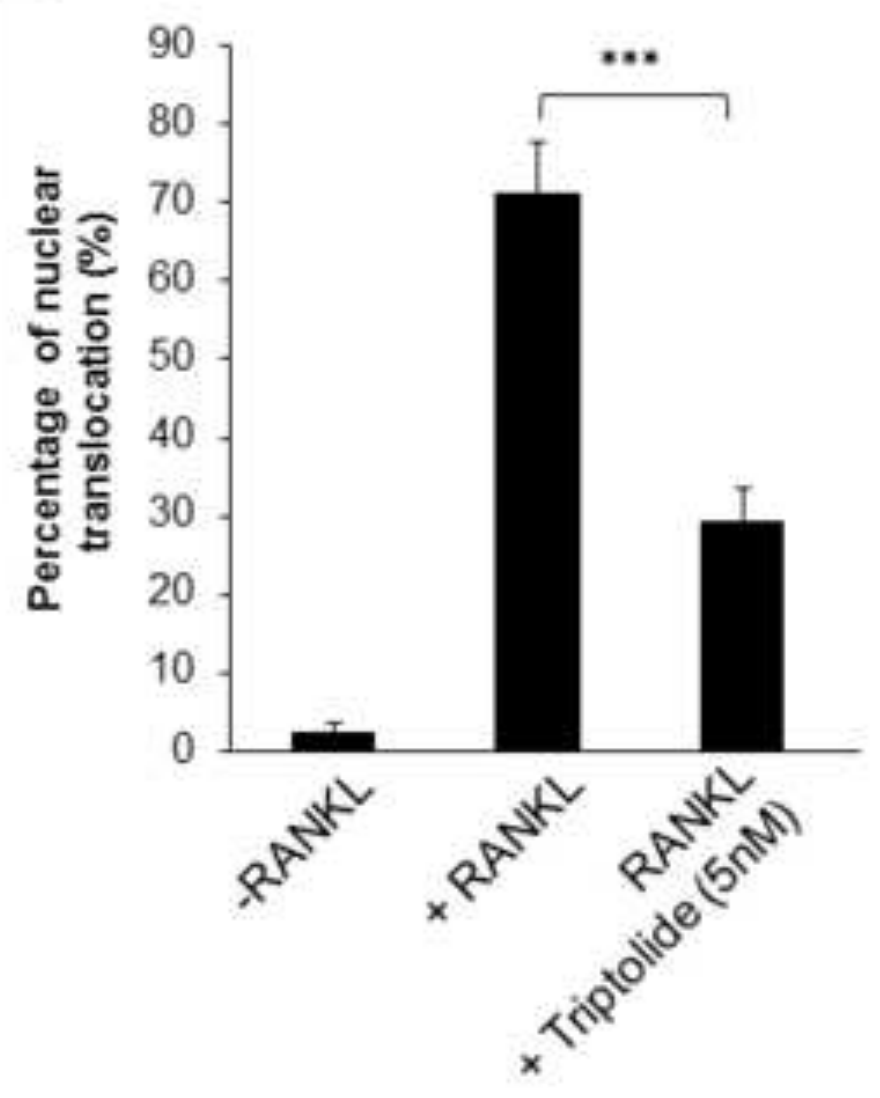

Fig. 5 
A

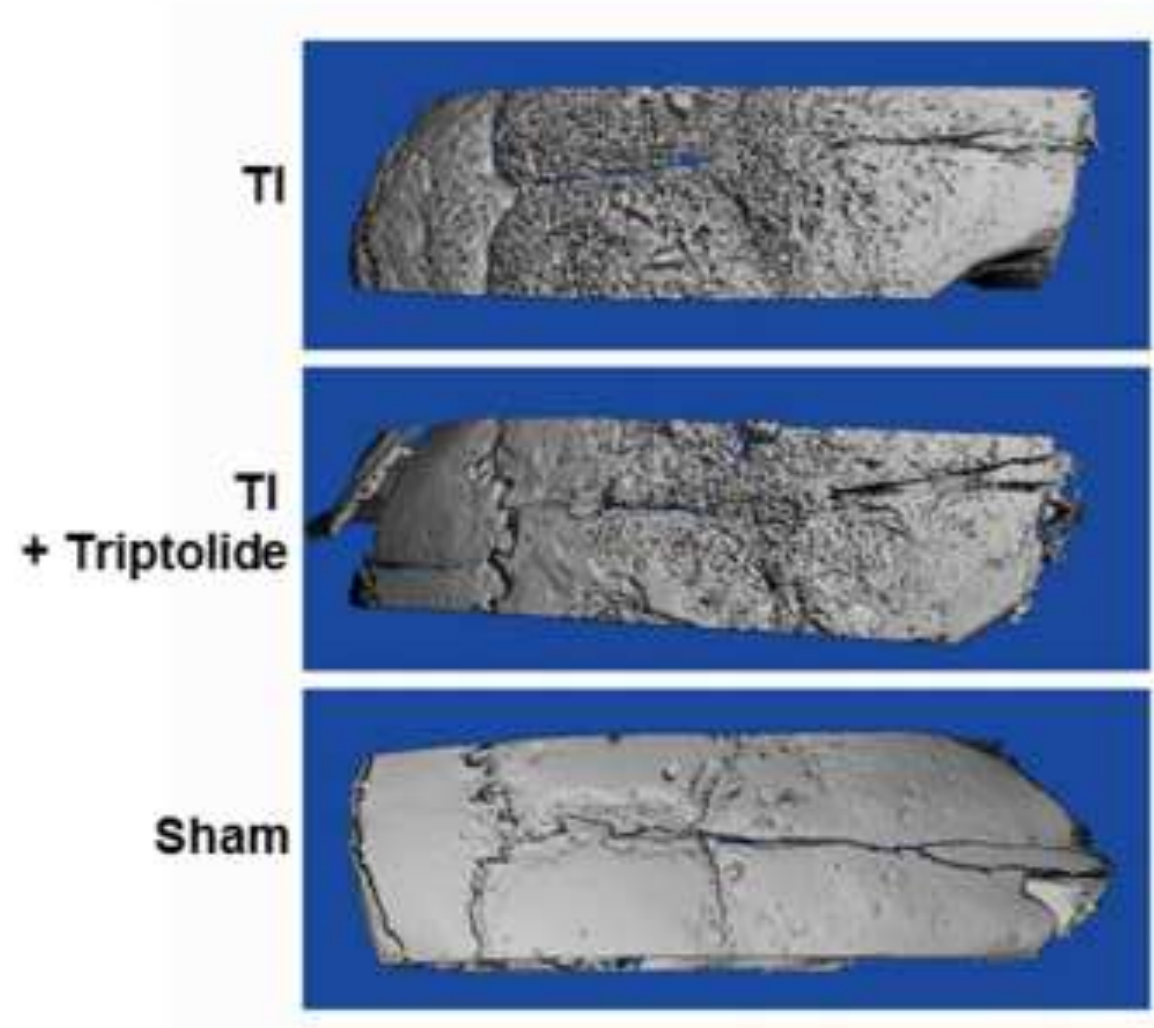

B

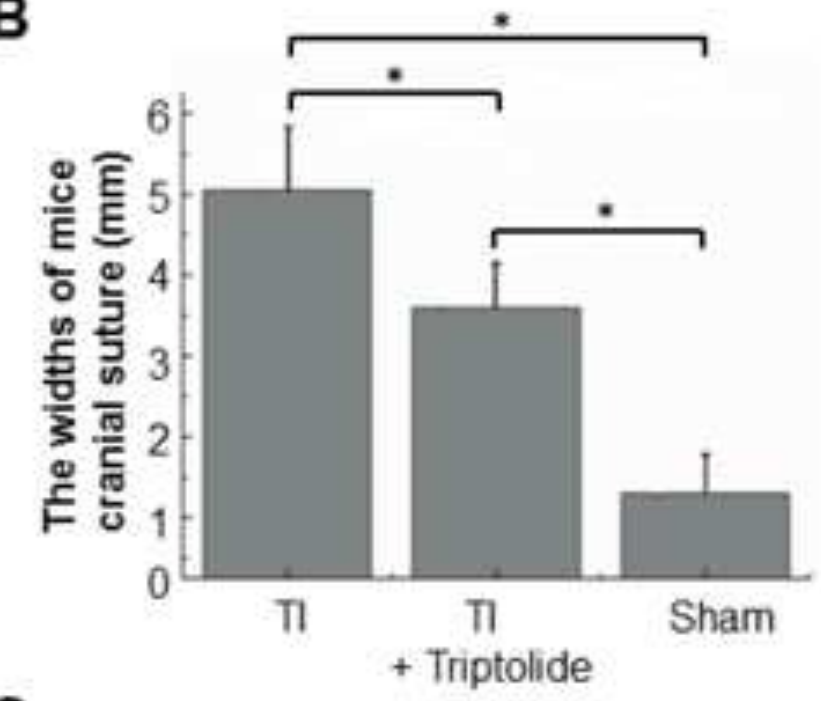

C

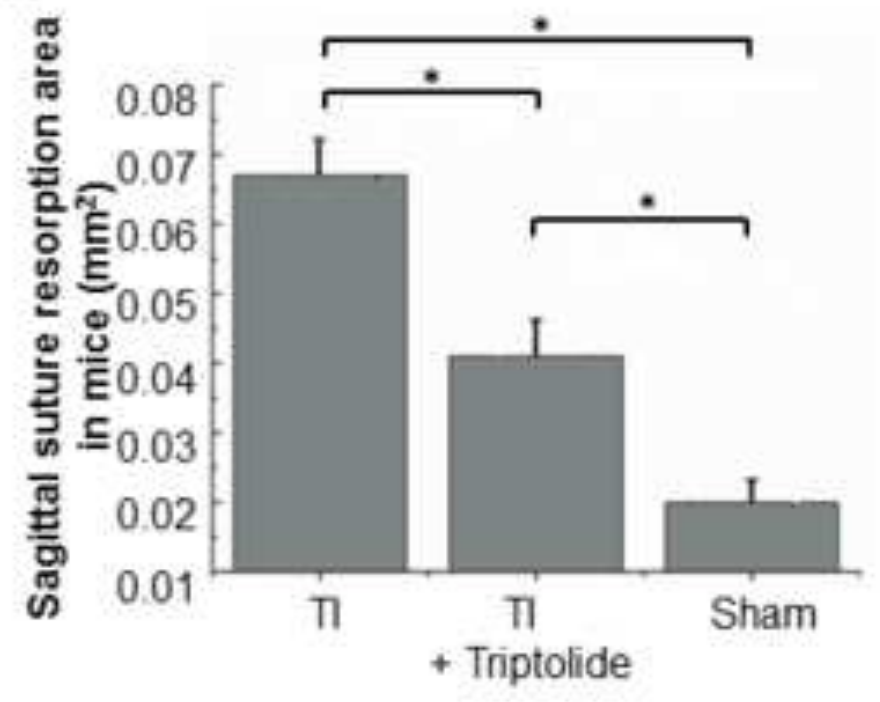

Fig. 6 